\title{
Le 'cas' littéraire du manuscrit L.III.17 de la «Biblioteca Nazionale Universitaria» de Turin: une traduction ambitieuse
}

\section{Stefania Vignali}

\section{(2) OpenEdition}

Journals

Édition électronique

URL : http://journals.openedition.org/studifrancesi/693

DOI : 10.4000/studifrancesi.693

ISSN : 2427-5856

Éditeur

Rosenberg \& Sellier

Édition imprimée

Date de publication : 1 août 2015

Pagination : 223-234

ISSN : 0039-2944

Référence électronique

Stefania Vignali, «Le 'cas' littéraire du manuscrit L.III. 17 de la «Biblioteca Nazionale Universitaria» de Turin: une traduction ambitieuse », Studi Francesi [En ligne], 176 (LIX | II) | 2015, mis en ligne le 01 août 2016, consulté le 18 septembre 2020. URL : http://journals.openedition.org/studifrancesi/693 ; DOI : https://doi.org/10.4000/studifrancesi.693

\section{(c) $(1)$}

Studi Francesi è distribuita con Licenza Creative Commons Attribuzione - Non commerciale - Non opere derivate 4.0 Internazionale. 


\title{
Le 'cas' littéraire du manuscrit L.III.17 \\ de la «Biblioteca Nazionale Universitaria» de Turin: une traduction ambitieuse
}

\begin{abstract}
This study is focused on the earliest French translation of Dante's Inferno, which is contained in the L.III.17 bilingual manuscript from the Turin National Library. Little is known with certainty about the context of this unique work, so we try to investigate it as an archaeological artifact and start the analysis from its peculiar codicological aspects, as compared to other prior and contemporary bilingual manuscripts.

The choice of the hendecasyllabic verse and humanist calligraphy for the Italian source, and of the alexandrin verse and the bastard calligraphy for the French version, the specular text layout, the relevant and, probably, authorial corrections (showing extreme attention to the curation of the text, unfortunately incomplete): all these aspects contribute to shed light on the underlying, almost avant-garde for the times, ideology that the raising French language deserve the same prestige as the authoritative Tuscan model, and finally has the possibility to detach from it, compete with it, and live on its own.
\end{abstract}

Le manuscrit L.III.17 de la Biblioteca Nazionale Universitaria de Turin est un volume modeste à l'apparence, bien différent des exemplaires de luxe destinés aux personnalités illustres. Incomplet et partiellement endommagé par l'incendie de $1904^{1}$, à l'état actuel il est dépourvu de préface et de colophon ${ }^{2}$, ainsi que de marques de possession ${ }^{3}$ : toute information concernant le destinataire, le copiste, l'auteur des dessins à la plume qu'il conserve est donc absente. Même l'époque à laquelle il entra dans les collections ducales reste obscure.

Les recherches menées jusqu'à maintenant ont essayé de fournir des réponses à quelques-unes de ces questions: pour ce qui est du milieu dans lequel ce codex vit le jour, nous avons suggéré ailleurs des liens possibles avec l'entourage de Jean Lemaire

(1) Sur l'incendie de la Biblioteca Nazionale Universitaria de Turin voir G. GORRINI, L'Incendio della Biblioteca Nazionale di Torino, Torino-Genova, Renzo Streglio \& C $C^{\text {ia }}, 1904$.

(2) On ne pourrait tout de même exclure à priori leur existence à l'époque de la constitution du codex. En effet, l'aspect actuel du manuscrit nous suggère qu'il dut passer à travers beaucoup de vicissitudes.

(3) Parfois, sur le blanc des pages, on constate la présence d'interventions d'autres mains. Il s'agit le plus souvent d'essais de plume, rédigés dans une écriture cursive très peu soignée, et sans doute postérieurs à la rédaction du codex.

(4) Sur cette question nous nous permettons de renvoyer à notre contribution $\grave{A}$ propos de la pre- mière traduction de l'Enfer" de Dante: un modèle poétique? en cours de publication dans les Actes du Colloque Medieval Translator 2013.

(5) Cette hypothèse se fonde principalement sur l'identification du texte italien copié dans le manuscrit, qui coïnciderait avec le texte publié à Venise par les soins de Pietro De Piasi, dit le Cremonese, en 1491. Sur cette question voir J. CAMus, La première version française de l'Enfer de Dante, in «Giornale Storico della letteratura italiana», XXXVII (1901), pp. 70-93 et A. BerTolino, A propos de la première traduction de l'Enfer de Dante (Turin, BNU, L. III. 17): quelques questions préliminaires, «Le Moyen Français», 71 (2012), pp. 3-32. 
de Belges ${ }^{4}$; quant à l'époque de sa confection, il a été établi que le terminus post quem pourrait être fixé à l'année $1491^{5}$.

Malgré la rareté des informations, le contenu du codex ne saurait justifier le désintérêt de la critique moderne à son égard': il s'agit en effet de l'unique témoin de la toute première traduction de l'Enfer de Dante en langue française et il revêt un rôle essentiel dans la reconsidération de la réception française du poeta theologus, perçue encore aujourd'hui comme l' «histoire d'une absence» ${ }^{7}$. Nous ne nous attarderons pas sur les raisons pour lesquelles Dante, contrairement à Pétrarque et à Boccace, ne fut guère apprécié par le public français des $\mathrm{XIV}^{\mathrm{e}}$ et $\mathrm{XV}^{\mathrm{e}}$ siècles, ces aspects ayant déjà été éclairés ${ }^{8}$. Nous croyons néanmoins que l'originalité qui caractérise cet ouvrage n'a pas été suffisamment assez mise en lumière.

Parmi les érudits qui se sont occupés du texte contenu dans notre manuscrit, ${ }^{9}$ plusieurs ont souligné la singularité des choix stylistiques du traducteur, qui non seulement a adopté la technique de la traduction ad verbum, dans la perspective de la plus parfaite correspondance avec l'original italien, exceptionnellement présent dans le manuscrit, mais qui a aussi choisi de transposer systématiquement l'hendécasyllabe italien en alexandrins et surtout de reproduire le schéma rimique de la 'terza rima'"

À une époque où le débat autour de la langue vernaculaire est ardent, ces choix de traduction nous semblent surtout l'expression de la volonté de prouver, même par la mise en page du manuscrit, que le français et l'italien jouissent d'un même prestige.

(6) Cité à maintes reprises, le manuscrit n'a fait l'objet que de quelques publications partielles (C. SAlvioni, Il canto X dell' "Inferno" di Dante - Nozze Chicherio-Fanciola, Bellinzona, Tip. C. Salvioni, 1889; C. Casati, Fragments d'une ancienne traduction française de Dante, «Bibliothèque de l'École des chartes», XXV, 5 (1864), pp. 304-320), et d'une édition sans doute défaillante (C. MoreL, Les plus anciennes traductions françaises de la Divine Comédie, Paris, Librairie Universitaire, 1897). Une étude philologique partielle, mais encore sous forme de notes, a paru par les soins du philologue allemand E. STENGEL (Philologischer Kommentar zu der französ. Übertragung von Dantes Inferno in der Hs. L 3. 17 der Turiner Universitätsbibliotbek, Paris, H. Welter, 1897). Seul Jules Camus (art. cit.) et, plus récemment, Alessandro Bertolino (art. cit.), ont mené des recherches plus approfondies sur ce manuscrit.

Nous signalerons que celui-ci ne figure pas dans le répertoire Translations médiévales: cinq siècles de traductions en français au Moyen Âge (XI- $X V^{\bullet}$ siècles), (dir. C. GALDERISI, Turnhout, Brepols, 2011), puisqu'il est considéré un exemplaire remontant à une époque postérieure par rapport à celle prise en considération dans cet ouvrage. Il en est de même pour les manuscrits qui conservent la traduction des Triomphes de Pétrarque exécutée par Simon Bourgouin dont il sera question plus bas.

(7) J. Risset, Dante en France. Histoire d'une absence, dans L'Italia letteraria e l'Europa. Atti del Convegno internazionale di Aosta (20-23 ottobre 1997), a cura di N. Borsellino e B. Germano, Roma, Salerno, 2 voll., t. I, pp. 59-71. Voir aussi A. FARINELli, Dante e la Francia dall'età media al secolo di Voltaire, Milano, Hoepli, 1908.

(8) Cf. A. BerTolino, art. cit.; S. Vignali, À propos de la première traduction de l' "Enfer" de Dante: un modèle poétique? cit.
(9) Outre les études déjà citées nous signalerons encore: S. MAFFEI, Lettera del Sig. Marchese Scipione Maffei al Sig. Apostolo Zeno in data de' 26. Giugno del corrente anno da Torino, in «Giornale dei letterati d'Italia», VI (1711), pp. 449-484, spéc. pp. 474-475; P. ColomB DE Batines, Bibliografia dantesca, ossia Catalogo delle edizioni, traduzioni, codici manoscritti e comenti della Divina Commedia e delle opere minori di Dante, seguito dalla serie de' biografi di lui, compilata dal sig. visconte Colomb de Batines; traduzione italiana fatta sul manoscritto francese dell'autore, Prato, Tip. Aldina, 1845-1846, 3 voll., t. I, pp. 247248; E. LitTre, Dante, l' "Enfer" mis en vieux langage françois et en vers, Paris, Hachette, 1879 , pp. XXVIIXxxIV; K. WitTe, Dantiis Alligherii "Divina Comoedia" bexametris latinis reddita ab abbate Dalla Piazza vicentino, praefatus est et vitam Piazzae adiecit Karolus Witte, Lipsiae, I.A. Barth, 1848, pp. XXXIII-XXXIv; R. RENIER, Sulla più antica versione dal francese di Dante (Nozze Percopo-Santini): Nota di R. Renier, Torino, Vincenzo Bona, 1889.

(10) Ce choix fut audacieux vu que l'alexandrin ne devint le grand vers français que vers la fin du Xvi siècle. Cf. K. TogeBy, Histoire de l'alexandrin français, dans Études romanes dédiées à Andrea Blinkenberg, Copenhague, Munskgaard, 1963, pp. 240-266; M. R. Jung, L'alexandrin au XV siècle, dans Orbis medievalis. Mélanges de langue et de littérature offerts à Reto Bezzola, Berne, Franke, 1978, pp. 203-217; O. HalÉvy, La vie d'une forme: l'alexandrin renaissant (1452-1573), Thèse en Langue et littérature françaises soutenue à l'Université de Grenoble 3, 2003, 693 pp. De plus, si les hypothèses de datation sont correctes, notre traducteur serait le premier à avoir adopté le schéma de la terza rima en français, primauté qui pour l'instant revient à Jean Lemaire de Belges, qui l'adopta, en 1513, dans sa Description du temple de Venus. 
C'est pour cette raison que dans cette contribution, nous nous proposons d'analyser en premier lieu l'objet en soi, c'est-à-dire de traiter le manuscrit en tant qu'objet archéologique. En effet, fournir des informations codicologiques supplémentaires permettra de confirmer l'exceptionnalité, l'importance et l'originalité du codex et si ces données ne fournissent pas de réponses certaines à des questions encore ouvertes, elles pourront du moins suggérer d'autres pistes de recherche.

Quant à la description extérieure du codex, il s'agit d'un in-folio en papier qui se compose de $190^{11}$ feuillets mesurant en moyenne 310 x $205 \mathrm{~mm}$, auxquels s'ajoutent vingt-sept feuillets plus récents qui contiennent des annotations diverses dont la plupart concernent le sujet du manuscrit, mais qui remontent à une époque postérieure à sa composition ${ }^{12}$. Les feuillets apparaissent brûlés sur les côtés et sur le dos; le manuscrit est acéphale et la fin est absente, ce qui a été imputé jusqu'à maintenant aux dommages qu'il aurait subis durant l'incendie de $1904^{13}$. Toutefois, l'étude de Jules Camus, publiée en 1901, atteste qu'avant cette date, le manuscrit se composait du même nombre de feuillets ${ }^{14}$, et quelques années plus tôt, en 1897, Edmund Stengel affirmait: «Es ist eine Papierhs. von 190 einspaltigen Folio-Blattern zu je 30 Zeilen. Die Blatter 186, 188 und 190 sind zum grossen Teile zerstört» ${ }^{15}$. Et encore dans le catalogue rédigé par Bencini dans la première moitié du XVIII ${ }^{\mathrm{e}}$ siècle on trouve la description suivante: «Cod. Chart. -fol. 190 - in fol. L'Opera del Dante: in francese. Deficit in fine» ${ }^{16}$.

En outre, en 1889, Rodolfo Renier signalait que l'apparat iconographique était lui aussi incomplet:

Di questi disegni (che è inesatto chiamare miniature, come si fece, perché sono semplici disegni a penna e matita, con qualche ritocco di colore per dar risalto ai chiaroscuri) solo i primi cinque sono compiuti: degli altri non sussistono che delle inquadrature, ed anche le iniziali di ogni canto, che dovevano essere ornate, sono lasciate in bianco ${ }^{17}$.

En effet, la page contenant le début de chaque chant en français était destinée à accueillir des dessins à la plume, mais ceux-ci ne furent exécutés que pour les six premiers chants. Aujourd'hui, comme l'avait affirmé Renier, le manuscrit n'en conserve que cinq à cause de la disparition du $\mathrm{f}$. $7 \mathrm{r}$ qui devait préserver le dessin du deuxième chant et à la place duquel les restaurateurs ont introduit un feuillet blanc.

Ces attestations, toutes antécédentes à l'incendie de 1904, démontrent donc que le manuscrit se trouvait déjà dans le même état de conservation qu'aujourd'hui, à l'exception peut-être des brûlures sur les bords des feuillets, information sur laquelle toutefois tous ces spécialistes se taisent, mais qui auraient pu se produire aussi, si le manuscrit était arrivé plus tôt à Turin, lors de l'incendie qui avait eu lieu dans l'ancienne bibliothèque ducale en $1667^{18}$.

(11) L'ensemble du manuscrit présente une double numérotation, une première, plus ancienne, sur la marge droite en haut du feuillet qui indique la présence de 192 feuillets et une deuxième, plus moderne, écrite au crayon en bas de page, qui n'en compte que 190 (A. BERTOLino, art. cit., p. 8). Toutefois, d'autres signes situés sur le bord inférieur de la page aujourd'hui partiellement brûlés mais encore lisibles, ont été identifiés aux ff. 65r, 66r, 67r, 68r. Il s'agit de notations numériques, remontant vraisemblablement à l'époque de la composition du codex, grâce auxquelles il sera peut-être possible de reconstituer le nombre de cahiers dont le manuscrit se composait à l'origine.
(12) Cf. la description fournie par A. BERTOLINO, art. cit., pp. 8-9.

(13) Voir la note 1 précédente.

(14) J. Camus, art. cit., p. 72.

(15) E. STEngel, Philologischer Kommentar cit., p. 3.

(16) F.D. BENCINI, Indice de' libri Ebraici, Greci, Latini, Italiani e Francesi, IV, s.d.. Cf. aussi A. GIACCARIA, I fondi manoscritti della Biblioteca Nazionale Universitaria di Torino, in «Pluteus», 2 (1984), p. 182

(17) R. RENIER, op. cit., p. 9.

(18) A. GIACCARIA, art. cit., p. 177. 
Il est bien dommage qu'aucun d'entre eux n'ait ressenti la nécessité de nous fournir des indications au sujet de la reliure. En effet le travail de restauration auquel le codex fut soumis en $1909^{19}$ a pourvu le volume d'une reliure moderne; par conséquent les informations qu'on aurait pu tirer de l'aspect de la couverture, et qui auraient été très utiles pour préciser la date de confection du manuscrit, son milieu de production ou pour formuler des hypothèses sur l'identification du destinataire et des possesseurs, ont malheureusement été perdues à tout jamais.

En ce qui concerne la question de l'époque à laquelle le manuscrit fut confectionné, dans une étude récente Alessandro Bertolino confirme l'hypothèse déjà avancée par Jules Camus, qui le faisait remonter à une époque comprise entre 1491 et le début du XVI ${ }^{\text {e }}$ siècle. Leurs suppositions se fondent principalement sur l'identification du texte italien copié dans le manuscrit ${ }^{20}$, sur les dessins ${ }^{21}$ et surtout sur l'étude des deux filigranes présents aux ff. 129, 165, 170, 190, identifiés sur la base du catalogue de Briquet, auquel Camus n'avait pu avoir accès ${ }^{22}$. À ce propos, nous croyons nécessaire d'apporter ici une petite rectification: pour ce qui est du filigrane représentant le «pot à anse dont le couvercle porte une croix ordinaire» ${ }^{23}$ nous concordons aves les identifications possibles fournies par nos prédécesseurs; par contre, pour ce qui est de la «croix en forme de tau grec, surmonté d'un fleuron» ${ }^{24}$, Bertolino propose une ressemblance possible avec les numéros 5466, 5467 et 5569 du catalogue de Briquet $^{25}$. En réalité, pour sa forme et pour sa taille, même si la distance des vergeures ne semble pas coïncider parfaitement, il correspond au numéro 9125 du même catalogue, dont l'existence est attestée dans un seul exemplaire produit à Lubeck en 1530, aujourd'hui conservé aux Archives de la ville de Bremen ${ }^{26}$. Cette rectification nous permet de déterminer une fourchette temporelle plus précise quant à la date de production du papier sur lequel le manuscrit fut rédigé, qui peut être fixée vraisemblablement entre la fin du Xv $\mathrm{xv}^{\mathrm{e}}$ siècle ${ }^{27}$ et 1530.

Comme on l'a vu, la question de la datation du codex a été abordée principalement à l'aide d'une analyse directe du manuscrit; il est donc possible que d'autres informations puissent être repérées par le même procédé.

Le ms L.III.17 est l'un des rares exemples de codex bilingue en papier présentant sur le feuillet de gauche le texte italien écrit en humanistique ronde et sur le feuillet de droite la traduction française copiée en bâtarde.

Albert Derolez a donné la définition de ce qu'il entendait par manuscrit humanistique, caractérisé principalement par le type d'écriture; à ce propos il affirmait: «Tout d'abord il ne s'agit que de mss sur parchemin» ${ }^{28}$ et il poursuivait: «dans les collections des grands bibliophiles du Quattrocento, les manuscrits en

(19) Le codex fut restauré dans l'atelier Pachiotti en 1909, mais le mauvais état de conservation dans lequel le manuscrit se trouve aujourd'hui rend nécessaires et urgentes de nouvelles interventions pour le préserver. Cf. Mostra di recuperi e restauri, Torino febbraio-marzo 1986, Biblioteca Nazionale Universitaria. Mostra e catalogo a cura di Angelo GIACCARIA, pp. 24-25.

(20) Cf. la note 5 précédente.

(21) Sur ce sujet voir aussi l'étude de L. RENZI, Le conseguenze di un bacio, Roma, il Mulino, 2007, pp. 194-198.

(22) Ce catalogue, datant de 1923, est postérieur de 22 ans par rapport à l'ouvrage de Camus, publié en 1901. C.-M. BRIQueT, Les filigranes: Dictionnaire bistorique des marques du papier, Leipzig, Hiersemann, 1923 (aujourd'hui dispo- nible aussi sur le site http://www.ksbm.oeaw. ac.at/_scripts/php/BR.php).

(23) Cette description est tirée de J. Camus, art. cit., p. 72.

(24) Ibidem

(25) A. BerTolino, art. cit., p. 11.

(26) C.-M. BRIQUET, op. cit., t. III, p. 484.

(27) Les deux filigranes représentant le pot à une anse qui semblent correspondre à celui qui est conservé dans le codex turinois sont respectivement le n. 12499 (produit probablement entre 1492 et 1508 ) et le n. 12500 (dont l'existence est attestée vers 1518/1519) du catalogue de Briquet.

(28) A. Derolez, Codicologie des manuscrits en écriture bumanistique sur parchemin, Turnhout, Brepols, 1984, 2 voll., t. I, p. 11.

(29) Ibidem. 
papier sont extrêmement rares» ${ }^{29}$. Le papier, à son avis, ne convenait qu'aux mss personnels, aux collections de notes et de correspondance, aux livres destinés aux clercs pauvres et à ceux, en langue vulgaire, destinés à un public large, car ils sont moins soignés, souvent la réglure n'est pas complète, ils ne sont généralement pas décorés et ils présentent une écriture rapide. Ce savant soulignait encore que les collectionneurs n'apprécient guère les mss sur papier ${ }^{30}$. Cette tendance toutefois ne saurait être confirmée pour ce qui est de la bibliothèque des ducs de Savoie dont notre ms a pu faire partie ${ }^{31}$ : en effet, la consultation de l'inventaire des châteaux de Chambéry, Turin et Pont d'Ain rédigé en $1498^{32}$ témoigne de la présence d'un nombre considérable de volumes en papier. Or, même si le manuscrit dont il est question ici est copié sur papier, son aspect ne correspond qu'en partie à la description fournie par Derolez, en particulier pour ce qui est de la prétendue négligence de son exécution.

Nous avons donc préféré adopter l'attitude moins intransigeante d'Armando Petrucci, selon lequel notre manuscrit constitue un exemplaire de «libro umanistico» ${ }^{33}$, qui pouvait être écrit sur parchemin ou sur papier et qui était destiné à la bibliothèque des doctes ou de leurs protecteurs.

D'ailleurs, même s'il ne s'agit pas d'un volume luxueux, et bien que son état de conservation ne soit pas excellent, le lecteur ne peut que rester ébloui à son ouverture.

Sa mise en page respecte dans les détails les plus menus les critères de lisibilité du texte. Quatre lignes verticales et deux lignes horizontales signalent la présence d'une réglure des plus simples, symptomatique d'un choix esthétique intentionnel de clarté et simplicité. Le texte, positionné au centre de la page, est écrit sur une seule colonne et la page présente un grand équilibre entre espaces blancs et espace d'écriture. Tous les feuillets présentent aussi une réglure à l'encre brune, parfois à peine perceptible ${ }^{34}$, disposée pour accueillir trente vers tant pour la partie italienne que pour le texte français, à l'exception des feuillets réservés aux débuts des chants et destinés à recevoir six lignes de texte seulement.

Deux altérations à ce système sont toutefois à signaler: la première au f. $33 \mathrm{r}$, où la transcription du texte français s'interrompt brusquement après le v. 54 et laisse un vide de douze lignes, alors que le texte italien correspondant ne présente aucune interruption, ce qui fait penser que le texte italien devait déjà figurer dans le codex au moment de la copie du texte français et que le copiste de celui-ci a dû, à ce moment, percevoir une véritable incongruité dans l'équivalence des textes, à tel point qu'il s'est vu contraint d'abandonner la copie, qu'il n'a reprise qu'au feuillet successif ${ }^{35}$. Une deuxième altération figure aux ff $89 \mathrm{v}-90 \mathrm{r}$, où les deux textes présentent onze tercets au lieu des dix prévus; contrairement à ce qui s'est produit précédemment, à

(30) Ibid., p. 12-sqq.

(31) On ne saurait actuellement établir avec précision la date à laquelle notre manuscrit entra dans la bibliothèque ducale, même si sa présence est attestée avec certitude à partir de 1713. G. MACHET, Index alphabétique des livres qui se trouvent en la Bibliothèque Royale de Turin en cette année 1713. Sous le règne de S.M. Victor Amé, Roy de Sicile, et de Chipre, Duc de Savoye, et de Monferrat, Prince de Piemont, 2 voll., s.d., t. II, p. 693.

(32) P. VAYRA, Le lettere e le arti alla corte di Savoia nel secolo XV. Inventari dei castelli di Ciamberi, di Torino e di Ponte d'Ain, 1497-1498, Torino, Stamperia reale della ditta G.B. Paravia e C. di I. Vigliardi, 1883.

(33) A. Petrucci, Alle origini del libro moderno, in «Italia medioevale e Umanistica», XII (1969), p. 297.
(34) En effet, l'encre s'est dissoute à plusieurs endroits et de nombreuses moisissures ont fait leur apparition. Heureusement le texte demeure encore bien lisible.

(35) Nous avons traité cette question dans S. VIGNALI, De quelle source peut-on parler pour la première traduction de l'Enfer" de Dante?, à paraître dans Nature et définition de la source, éd. M. Maulu, Chemins de tr@verse, («Chemins Italiques»). Voir aussi A. BERTOLINO, art. cit., p. 19 .

(36) Ce chiffre ne saurait passer inaperçu à un lecteur de Dante, qui attribuait une valeur fondamentale à la numérologie. Le chiffre trente, respecté pour la quasi-totalité du texte, ne nous semble donc pas un hasard. 
la fin de chaque feuillet un tercet manquant a été ajouté tant dans le texte italien que dans la version française ${ }^{36}$.

La réglure a été exécutée pour la totalité du manuscrit et bien que les mesures de la hauteur des lignes qui la composent varient entre $0,7 \mathrm{~cm}$ et $1,1 \mathrm{~cm}$, l'esthétique générale de la page n'en est pas compromise.

Le critère de lisibilité est aussi respecté grâce à l'emploi très parcimonieux de signes d'abréviation, qui ne dérangent aucunement le confort de lecture tant pour le texte italien que pour le texte français ${ }^{37}$; de plus, l'écriture de type «below top line», qui se définit par le non-usage de la ligne de tête de la réglure, de manière à ce que le texte apparaisse à l'intérieur d'un cadre, aide à conférer à la page un aspect très équilibré38.

Cependant, malgré le soin porté à la clarté de son exécution, plusieurs indices nous suggèrent que notre manuscrit ne peut être considéré une copie définitive. Déjà en 1897, Edmund Stengel affirmait: «Der französische Text ist nicht [...] allseitig angenommen zu sein scheint, von Anfang bis zu Ende von ein und derselben Hand geschrieben» ${ }^{39}$. L'examen paléographique du texte français permet en effet d'identifier la présence de plusieurs mains qui s'alternèrent dans l'exécution de la copie, plus spécialement à partir du f. 155r. Mais, si dans certains cas le changement de main pourrait être mis en doute et justifié par une coupe différente de la plume, une rupture du corpus calligraphique est bien plus évidente au f. $164 \mathrm{r}$, et à partir du f. $175 \mathrm{r}$ on perçoit clairement un nouveau changement dans la calligraphie ${ }^{40}$. Enfin, dans les derniers feuillets (du f. 188r au f. 190r) une autre main intervient, dont le trait semblerait très proche de celui qui a rédigé la première partie de la copie du texte français. On compte donc l'intervention d'au moins trois mains dans la confection du texte français présent dans le manuscript.

Les textes français et italien firent aussi l'objet de repentirs et de corrections exécutés tant au moment de la confection du codex, qu'après coup ${ }^{41}$. Pour ce qui est du texte italien, vraisemblablement copié par une seule main, il fut l'objet de corrections visant essentiellement au rétablissement de sa correspondance au texte de la traduction française, principe fondamental auquel la traduction est soumise. Les interventions, qui concernent plus particulièrement le lexique, peuvent être surchargées au mot préexistant ou interlinéaires; les premières furent exécutées vraisemblablement par la même main qui rédigea l'ensemble du texte (ex. per altre porte $>$ per altra port $a, f .9 \mathrm{v})$, tandis que les deuxièmes furent apportées au texte par une autre main (ex. La vita > l'adiuta, f. 15v).

Au contraire, plusieurs genres de corrections figurent dans le texte français: il peut s'agir de corrections sur-écrites à la première rédaction dans le but de rétablir l'orthographe d'un mot considérée plus «correcte» (par exemple toy sur to $i$ au f. $4 \mathrm{r}$ ) et elles sont alors généralement de la main du copiste. D’autres révisions, qui se signalent par l'emploi d'une encre différente, furent exécutées après la rédaction primitive: elles peuvent concerner l'insertion de monosyllabes afin de rétablir la mesure

(37) La plupart des signes concernent l'emploi du tilde sur la voyelle pour marquer l'abréviation de la nasale. Les autres cas sont très rares.

(38) Sur l'écriture «below top line» cf. A. DEROLEZ, op. cit., t. I, pp. 83-84; N.R. KERR, From 'above top line' to 'below top line': a change in scribal practice, «Celtica», V (1960), pp. 13-16; J. VezIN, La réalisation matérielle des manuscrits latins pendant le baut Moyen Âge, dans Codicologica, t. II, Éléments pour une codicologie comparée, Leyde, E.J. Brill, 1978 («Litterae textuales»), pp. 15-51, p. 34.

(39) E. STENGEL, Philologischer Kommentar cit., p. 5.
(40) Elle se présente avec des hastes et des hampes beaucoup plus prononcées et, par exemple dans le cas de l'exécution de la lettre -d, un trait très «renaissant».

(41) Cf. A. Bertolino, art. cit., pp. 19-20; S. VIGNALI, À propos de la première traduction cit.

(42) Le mot escorte (< it. scorta), attesté dans les dictionnaires du XVI ${ }^{\mathrm{e}}$ siècle, dut prendre en français le même sens général qu'il avait en italien, mais il acquit de plus en plus le sens de «conduite militaire». Voir E. Huguet, Dictionnaire de la langue française du seizième siècle, Paris, Champion, 1925-1967, 
de l'alexandrin (ex. f. 11r: «Hor a besoing de toy \ce/ pauvre jouvencel»), ou de

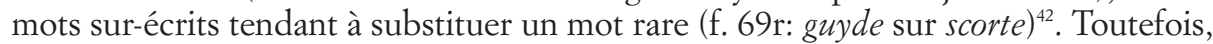
ces corrections ne semblent pas abîmer la mise en page du texte, qui demeure assez propre. Par contre, une troisième typologie de corrections, portant spécifiquement sur des modifications apportées à la substance même de la traduction, a sans doute une implication plus importante, en particulier pour ce qui est de la détermination de l'autographie du codex ${ }^{43}$.

Le cas le plus évident figure au treizième chant (f. 75r). Dans la version originale on lit les vers suivants:

Icy les trainerons, et par le long de ceste

Triste forest seront les nostres corps pendus,

Ung chescun au prunier de son umbre moleste.

Au tronc avions encor nos visaiges tendus,

Cuidant qu'il nous voulust quelque aultre chose dire,

Quant par nous furent telz buz et criz entendus,

Comme qua[n] t droit au lieu ou s'est voulu reduyre

Le chasseur sent venir porc ou beste sauvage [...]

Une main différente a corrigé le texte de la façon suivante:

Icy les trainerons, et seront parmy ceste

Triste forest pendus nos corps vains, sans esperitz,

Ung chescun au prunier de son umbre moleste.

Nous estions vers le tronc anchores ententiz,

Cuidant qu'il nous voulust quelque aultre chose dire,

Quant fumes de ung rumeur et de tel bruit surpriz,

Que une garde entent, lors que venir et reduyre

Sent le cerf à sa poste, ou le porceau sauvage [...]

Ces modifications ont été apportées avec une encre et une écriture cursive différente par rapport à la totalité du texte, et elles concernent des aspects qui ont à faire avec les mots à la rime, l'ordre des membres de la phrase, les choix lexicaux; il s'agit donc d'interventions qui peuvent être attribuées soit à l'auteur même de la traduction, soit à un relecteur très attentif ou à un réviseur; en effet, il est connu que certains traducteurs, dont Lemaire de Belges, avaient l'habitude d'adopter une telle attitude ${ }^{44}$. Ces aspects nous semblent aussi symptomatiques du changement de destination du manuscrit, qui fut conçu au début pour être offert et qui devint, au cours de son élaboration, une copie de travail; nous reviendrons plus loin sur cette question.

Cependant, déterminer ou refuser l'autographie d'un texte ne peut tout de même pas s'appuyer sur la seule étude paléographique; une telle étude doit être supportée

7 voll., s.v. escorte 1; B.H. WIND, Les mots italiens introduits an français au XVI siecle, Utrecht, HES Publishers, 1973, pp. 127-128.

(43) Sur cette question cf. O. Delsaux, T. van HEMELRYCK, Les manuscrits autographes en français au Moyen Age. Guide de recherche. Avec trois articles de G. Ouy, Turnhout, Brepols, 2014 («Texte, codex \& contexte», 5).

(44) Cf. A. Schoysman, Jean Lemaire de Belges et la Généalogie d'Anne de la Tour d'Auvergne dans le ms. 74 G 11 de La Haye (1518), «Le Moyen Français», 57-58 (2005-2006), pp. 325-326.
(45) G. OuY, Manuscrits autographes en France au temps des premiers bumanistes, dans Gli autografi medievali. Problemi paleografici e filologici. Atti del Convegno di studio della Fondazione Ezio Franceschini (Erice, 25 settembre-2 ottobre 1990), a cura di P. Chiesa e L. Pinelli, Spoleto, Centro italiano di Studi sull'Alto Medioevo, 1994, pp. 269-305; G. PARussa et R. TRACHSLER, 'Or sus, alons ou champ des escriptures'. Encore sur l'orthographe de Christine de Pizan: l'intérêt des grands corpus, dans Contexte and Continuities. Proceedings of the IV International Colloquium on Christine de Pizan (Glasgow 21-27 July 2000) published 
par une analyse des habitudes orthographiques du scribe, comme G. Ouy, puis G. Parussa et $\mathrm{R}$. Trachsler l'ont bien démontré ${ }^{45}$.

Nous avons donc mené des recherches supplémentaires afin de vérifier l'existence d'habitudes orthographiques récurrentes chez les différentes mains, mais elles n'ont malheureusement pas fourni d'indices probants. Si un certain souci de correction orthographique semble appartenir au premier copiste, par exemple dans l'usage généralisé de -i pour -jà l'initiale et à l'intérieur des mots, il nous a été impossible pour l'instant de déceler un système orthographique récurrent, chaque copiste orthographiant de manière différente le même mot à quelques lignes de distance; c'est le cas par exemple des alternances comme ombre (f. 21r) | umbre (f. 22r), voir (f. 55r) I veoir (f. 56r), peult (f. 181r) I peut (f. 182r), blaphemant (f. 185r) I blasphemant (f. 186r) ${ }^{46}$.

Même si la prudence reste de rigueur, la pluralité des mains qui ont copié le codex semblerait exclure la possibilité qu'il puisse s'agir d'un manuscrit autographe; néanmoins, la présence de modifications concernant des changements au niveau de la traduction pourrait signifier que sa manufacture est autographe, et de ce fait il pourrait être considéré comme autorial ${ }^{47}$.

Dans ce cas spécifique, il pourrait alors s'agir d'une rédaction de second jet prête à être copiée sur parchemin pour être offerte, ou préparée éventuellement en vue d'une diffusion par l'imprimerie ${ }^{48}$. En effet, le ms L.III.17 est considéré un manuscrit unique, mais l'on sait qu'en 1496 la bibliothèque de Charles d'Angoulême à Cognac conservait un «libvre de Dan, escript en parchemin et à la main, et en italien et en françois, couvert de drap de soye broché d'or, auquel il y a deux fermoers d'argent aux armes de feu mon dict seigneur; le quel libvre est historié» ${ }^{49}$. Ce volume est aujourd'hui introuvable; toutefois la mention du catalogue confirme qu'il ne s'agit pas de notre manuscrit en raison du support différent sur lequel il fut écrit. D'autre part, cela ne nous permet pas d'exclure à priori la possibilité d'une certaine parenté entre les deux codex, surtout pour la rareté de la coprésence du texte français et du texte italien dans un même manuscrit.

Dans ce cas, deux hypothèses pourraient être formulées au sujet de l'exemplaire turinois: si l'on considérait notre codex comme un manuscrit de second jet, alors il serait possible de penser que le ms. sur parchemin de Cognac aurait pu être une mise au net de celui-ci destinée à être offerte ${ }^{50}$; toutefois, cela nous paraît peu probable, à cause des filigranes suggérant une date de composition du codex turinois postérieure à l'année 1496. Le manuscrit turinois pourrait également être une copie du manuscrit de Cognac, mais cela paraît tout aussi invraisemblable en raison de la présence de nombreuses corrections; dans ce cas quelqu'un aurait été chargé de contrôler la traduction, ce qui expliquerait les rectifications et le 'déclassement' du codex au rang de copie de travail.

Quant à sa destination, tous ces aspects nous font supposer qu'il devait s'agir au début d'un manuscrit vraisemblablement confectionné pour un personnage illustre, italien ou italianisant, passionné de littérature italienne et peut-être aussi de Dante; toutefois,

in honour of Liliane Dulac, ed. by A.J. KENNEDY with R. Brown-Grant, J.C. Laidlaw et al., Glasgow, University of Glasgow, 2002, 3 voll., t. III, pp. 621-643.

(46) Il faut aussi souligner que l'activité de correction de la copie s'arrête brusquement à la fin du vingt-deuxième chant et donc il est possible aussi qu'une révision globale du texte aurait permis au réviseur d'intervenir sur ces aspects.

(47) Cf. la nomenclature dans O. Delsaux, T. VAN HEMELRYCK, op. cit., p. 7.

(48) Cf. G. Ouy, Manuscrits autographes cit., pp. 290-294; le lecteur pourra lire cette contribution aussi dans O. Delsaux, T. van Hemelryck, $o p$. cit., pp. 157-195. Cf. aussi O. Delsaux, Manuscrits et pratiques autographes chez les écrivains français de la fin du Moyen Âge. L'exemple de Christine de Pizan, Genève, Droz, 2013 («Publications romanes et françaises», 258), en particulier pp. 185-199.

(49) E. SÉNÉMAUd, La bibliothèque de Charles d'Orléans, comte d'Angoulême, en 1496, Paris, A. Claudin, 1861, p. 19.

(50) L'époque de l'arrivée de ce manuscrit à Turin n'est pas certaine. Quelques recherches menées dans cette direction suggèrent qu'il dut arriver à une époque comprise entre 1498 et 1659 . Cette question fera l'objet d'une étude à venir.

(51) En effet, à partir du vingt-deuxième chant tout travail de correction s'arrête. 
devenu une copie de travail au cours de son élaboration ${ }^{51}$, il dut avoir une diffusion très limitée, sinon nulle.

La destination première du codex justifie le choix très particulier de la mise en page qui, nous le rappelons, présente le texte original en regard de sa traduction française. Ici, le dessein idéologique propre au traducteur, qui doit refléter aussi les goûts de son destinataire, trouve sa parfaite correspondance dans l'aspect matériel du manuscrit.

Comme on le sait, l'existence de la traduction accompagnée du texte source ${ }^{52}$ dans le même manuscrit n'est pas pratique courante à la fin du Xve siècle ${ }^{53}$. Stefania Marzano a fourni une courte liste de ce genre de manuscrits ${ }^{54}$ : elle en cite deux du De Senectute, dans la traduction de Laurent de Premierfait ${ }^{55}$, un exemplaire de la traduction des Facta et Dicta memorabilia de Valère Maxime, tandis que pour ce qui est de la traduction du De amicitia elle souligne que si le prologue annonçait ce genre de disposition, aucun ms. bilingue ne nous est parvenu ${ }^{56}$. À ces rares témoins doivent être ajoutés ceux qui ont fait l'objet des recherches de Geneviève Hasenhor, dans le cadre d'une étude consacrée à la pratique des copistes médiévaux et aux problèmes qu'ils devaient aborder lorsqu'il s'agissait de prévoir la disposition de deux textes sur la page $^{57}$. Parmi les manuscrits sur lesquels elle a fondé son travail, tous remontant aux $\mathrm{XIII}^{\mathrm{e}}$ et $\mathrm{XIV}^{\mathrm{e}}$ siècles, figurent le rare exemplaire de luxe bilingue intitulé Dernière partie de la compilation historique présentée à Philippe le Long, la traduction des Disticha Catonis de Jean Le Fèvre, celle d'un poème de Pierre d'Eboli par Richard d'Eudes, les Proverbia magistri Serlonis, la traduction de la Cité de Dieu de Jean Chevrot, celle des Flores de civitate Dei de François de Meyronnes et surtout plusieurs manuscrits bilingues de deux des huit traductions du De consolatione philosophiae, à savoir neuf sur vingt-deux manuscrits de la traduction de Jean de Meun et onze manuscrits sur soixante-quatorze conservant la traduction anonyme, en vers et en prose, qui lui a été attribuée à tort ${ }^{58}$. À cette liste nous pouvons aujourd'hui ajouter un manuscrit de Jean Miélot découvert récemment ${ }^{59}$ et trois manuscrits qui conservent la traduction des Triomphes de Pétrarque réalisée par Simon Bourgouin au tout début du XVI siècle.

Comme Geneviève Hasenhor l'a montré, la présentation des deux textes en visà-vis a posé des problèmes spécifiques aux scribes: la traduction française étant tou-

(52) Comme l'a très bien souligné Giuseppe Di Stefano, identifier le texte source à partir duquel la traduction a été exécutée est fondamental afin d'en garantir la justesse et l'authenticité (G. Di STEFANO, L'edizione delle traduzioni: l'esempio del Decameron tradotto da Laurent de Premierfait (1414), dans Ensi firent li ancessor. Mélanges de philologie médiévale offerts à Marc-René Jung, publ. par L. Rossi avec la collab. de CH. JACOB-HugAn et U. BÄHLER, Alessandria, Ed. Dell'Orso, 1996, 2 voll., t. II, pp. 574 575). Toutefois, l'existence du texte original en regard de la traduction ne prouve pas nécessairement qu'il constitue sa source directe et des investigations supplémentaires dans cette direction doivent toujours être envisageés.

(53) Cette pratique se répandra plus tard dans les œuvres imprimées destinées pour la plupart à l'enseignement des langues étrangères. Cf. N. BINGEN, Le Maître italien, 1510-1660: bibliographie des ouvrages d'enseignement de la langue italienne destinés au public de langue française suivie d'un répertoire des ouvrages bilingues imprimés dans les pays de langue française, Bruxelles, E. Van Balberghe, 1987.
(54) S. Marzano, Laurent de Premierfait: entre le latin et le français, dans L'écrit et le manuscrit à la fin du Moyen Âge, éd. T. van Hemelryck et C. van HoOrebeEck, Turnhout, Brepols, 2006 («Texte, codex et contexte», 1), pp. 229-238.

(55) La traduction est conservée dans le ms. Paris, $\mathrm{BnF}$ lat. 7789, le manuscrit de Milan n'étant que sa copie. Le codex, qui présente une réglure très simple comme dans notre cas, s'ouvre avec le texte latin qui occupe les ff de $1 \mathrm{r}$ à $33 \mathrm{v}$; le texte français occupe la section qui va du f. $34 \mathrm{r}$ au f. $104 \mathrm{r}$. Ici la disproportion entre le texte latin et la traduction française est manifeste et la possibilité de proposer les deux textes en vis-à-vis dut paraître au copiste comme irréalisable.

(56) S. Marzano, op. cit., p. 233.

(57) Cf. G. Hasenhor, Discours vernaculaires et autorités latines, dans Mise en page et mise en texte $d u$ livre manuscrit, dir. H.-J. Martin et J. Vezin, Paris, Promodis, 1990, pp. 289-315.

(58) Ibid., p. 311.

(59) Cette découverte d'Elisabetta Barale, fait l'objet d'une contribution parue dans le n. 175 des «Studi Francesi», pp 63-74. Nous la remercions de nous avoir fait part de cette information. 
jours plus abondante que la prose originale, la difficulté majeure pour les copistes était celle de calibrer la mise en page de manière à faire coexister deux unités textuelles de longueur variée, tout en représentant sur la page la frontière linguistique des deux langues. Les stratégies adoptées furent très différentes: certains scribes jouèrent sur les effets de la dichromie, ou sur l'emploi de l'iconographie et de la position de la glose, d'autres firent recours à l'insertion d'une vedette annonçant le texte «Latin» ou «Français», à la disposition en enfilade ou enchaînée, à l'adoption d'une taille ou d'un module d'écriture différent. Il est aisé de constater que, dans ce genre de manuscrits, la plupart du temps le texte et sa source partagent la même feuille ${ }^{60}$; cette disposition permettait au lecteur d'avoir accès indifféremment au texte original et à sa traduction. Pour certains d'entre eux, le texte traduit avait donc la fonction de fournir une aide à la lecture de l'original, pour d'autres, la traduction était au premier plan ${ }^{61}$. Toutefois, dans presque tous les manuscrits cités ci-dessus, le texte traduit occupe la place et les fonctions normalement réservées à la glose et les copistes ne semblent pas avoir eu l'intention de le mettre sur le même plan que l'original. On remarquera encore que la plupart des manuscrits bilingues connus présentent des traductions latin-français et même si leur corpus est sans doute plus riche par rapport à celui que l'on a réuni actuellement, la pratique de présenter les deux textes dans le même manuscrit reste assez exceptionnelle.

Les manuscrits bilingues conservant des traductions de l'italien semblent être encore plus rares, mais ils sont particulièrement intéressants à cause des textes source choisis pour faire l'objet d'une traduction et de la perception du prestige dont jouissaient les langues italienne et française. Il faut avouer qu'à cette époque, ces traductions ne sont pas très nombreuses. De plus, le choix de traduire par la prose un antécédent en vers était encore perçu comme presque obligé ${ }^{62}$. La traduction des Triomphes de Pétrarque par Simon Bourguoin ${ }^{63}$ et celle de l'Enfer qui nous occupe ici, exécutées vraisemblablement à la même époque, furent les premières à utiliser le vers $^{64}$.

Des cinq manuscrits qui conservent la traduction des Triumphi par Bourgouin (vers 1501), trois présentent le texte italien en regard de la traduction française ${ }^{65}$. Le ms Paris, BnF fr. 12423, probablement composé pour le duc de Lorraine, est un exemplaire de luxe sur parchemin. Comme pour la plupart des manuscrits bilingues médiévaux, ici les deux textes, italien et français, coexistent sur la même page. Pour marquer l'articulation des divers éléments, le copiste a joué principalement sur la dichromie (brun pour les textes, rouge et bleu pour les titres, les titres courants et les devises, bleu, rouge et doré pour les lettrines) ${ }^{66}$ et il a adopté un module d'écriture particulier pour chacune des différentes parties du texte: l'humanistique italienne pour le français, la bâtarde pour le sommaire et une écriture italique de petit calibre

(60) Le choix de Laurent de Premierfait, dans sa traduction du De senectute, de proposer la transcription complète du texte latin avant le texte de la traduction, nous paraît tout de même assez singulier. Cf. aussi la note 55 .

(61) G. HASENHOR, op. cit., p. 315.

(62) Il suffit de consulter le répertoire Translations médiévales pour s'apercevoir de cette limite (Translations médiévales cit., pp. 1159-1173). Cf. dans le même volume la contribution de C. GALDERISI, Silences et fantômes de la 'translatio studii'. La traduction empêchée, t. I, pp. 433-457.

(63) Pétrarque, Les Triomphes. Traduction française de Simon Bourgouin, éd. crit. par G. PA-
Russa et E. SuOmela-Härmä, Genève, Droz, 2012. (64) Ibid., pp. 9-10. Pour la chronologie des traductions françaises des Triomphes pétrarquiens voir G. Parussa, I Trionfi di Petrarca tra l'Italia e la Francia: le metamorfosi di un testo, dans Atti del VII Congresso degli Italianisti Scandinavi, a cura di E. Garavelli e E. SuOmela-Härmä («Mémoires de la Société Néophilologique de Helsinki», LXVIII), Helsinki, 2005, pp. 71-87.

(65) Il existerait aussi un autre manuscrit bilingue conservant cette traduction, qui fait partie actuellement d'une collection privée. Cf. Pétrarque, Les Triomphes cit., p. 15 et pp. 70-71.

(66) Ibid., p. 62. 
pour le texte italien. Le texte français occupe le centre de la page, tandis que l'original italien est transcrit dans la marge gauche, en plus petit calibre, tant pour le recto que pour le verso des feuillets. Si le choix de cette mise en page semble s'inscrire dans le sillage de la tradition, il nous paraît évident que le copiste a attribué ici plus d'importance au texte français qu'au texte italien.

Bien plus singulière est la mise en page adoptée par le copiste des manuscrits BnF fr. 2500-250167. Pour différencier les deux langues, il a joué lui aussi sur la dichromie (bleu, rouge et or pour les titres des différentes parties, pour les incipit et les explicit $^{68}$, sur les lettrines et sur l'alternance de différents modules d'écriture (humanistique arrondie pour le français et italique pour l'italien). Mais contrairement au manuscrit précédent, le texte italien est transcrit sur la page de gauche en vis-à-vis du texte français, qui occupe le feuillet de droite.

Pour les copistes de ces manuscrits, la question la plus épineuse était la linéation de deux textes dont la longueur ne correspondait pas ${ }^{69}$; mais si le copiste du ms BnF fr. 12423 combla cet écart en choisissant de gloser le texte français à l'aide du texte italien, le copiste des mss BnF fr. 2500-2501 choisit plus astucieusement d'utiliser des lettrines, pour permettre au lecteur de se repérer et d'identifier plus aisément la correspondance de chaque section ${ }^{70}$.

Â propos du choix lié à la mise en page chez Bourgouin, Gabriella Parussa soulignait que «italiano e francese, se non hanno sempre la stessa dignità sulla pagina, sembrano coesistere nei manoscritti, come se il dialogo tra le due culture fosse ancora possibile [...]» $\gg^{71}$. Dans les manuscrits BnF fr. 2500-2501, il nous paraît évident que le copiste a eu l'intention de faire en sorte que texte source et traduction, et par conséquent langue italienne et langue française, soient mis sur un plan d'égalité et jouissent du même prestige.

L'analogie entre le choix de ce copiste et les stratégies de mise en page adoptées par celui qui a confectionné le ms BNT L.III.17 témoigne d'une intentionnalité et d'une maîtrise esthétique de la page tout à fait particulières. La lecture des manuscrits, qui peut se faire indistinctement dans les deux directions, montre clairement que l'intention du traducteur est celle de prouver que sa langue a finalement acquis un statut lui permettant de rivaliser avec le toscan, considéré jusqu'alors comme un modèle de langue à imiter et à assimiler, au même titre que les langues classiques. Le traducteur anonyme de l'Enfer pousse ces considérations à l'excès en choisissant de traduire presque ad verbum, ce qui lui permet non seulement de situer le français au même niveau que le toscan, mais de lui attribuer la même autorité ${ }^{72}$.

Le choix de proposer le texte italien en regard de la traduction n'est donc pas anodin. La présence de la source italienne dans le manuscrit permet en effet de vérifier la fidélité et l'exactitude philologique de la traduction, ce qui serait impossible autrement. Dans le cas du ms L.III.17, le choix de proposer le texte italien en regard

(67) «Il s'agit de deux manuscrits qui étaient à l'origine réunis avec un troisième contenant la première partie du texte et qui a disparu». Ibidem, p. 64.

(68) Ibid., p. 67

(69) En effet, le nombre des vers français est beaucoup plus important par rapport à celui dont se compose l'original. Cf. Ibid., p. 22.

(70) Des manuscrits conservant la seule traduction de Bourgouin seulement celui de la Beinecke Library de Yale (ms 875$)$ est en papier $(282 \times 200$ $\mathrm{mm}$ ); il est écrit à l'encre brune et il est dépourvu de toute décoration. Il s'agirait d'une copie plus tardive par rapport aux autres, certainement moins riche et moins soignée que les autres pour ce qui est de sa mise en page (seul le texte français est représenté), de l'écriture et de l'ornementation.

(71) G. Parussa, op. cit, pp. 79-80.

(72) Les progrès de la langue vulgaire se manifesteront peu après dans le choix d'exclure la présence du texte italien dans les manuscrits. En effet, le ms Arsenal 6480 et le ms conservé à Yale (Beinecke Library 875) conservent le seul texte français de la traduction de Bourgouin. Pour ce qui est des quatre traductions de la Commedia de Dante en français au cours du XVI siècle, à savoir celle du Paradis de François 
de sa traduction est d'autant plus intéressant que ce texte ne fut pas la source exacte à partir de laquelle la traduction fut exécutée $e^{73}$, ce qui nous pousse à nous interroger sur les raisons de sa présence dans le codex.

L'hypothèse la plus probable est que le texte italien fut donné à transcrire à un copiste qui n'était manifestement pas habitué à l'écriture humanistique ${ }^{74}$; il aurait donc utilisé comme spécimen le texte de l'Enfer tiré de l'édition vénitienne de 1491 publiée par les soins de Pietro De Piasi, dit le Cremonese. Il ne fait aucun doute que le choix de proposer l'humanistique pour le texte italien fut volontaire. En effet, ce genre d'écriture était généralement adopté pour les textes des auctoritates anciennes ${ }^{75}$, et son emploi permet donc au copiste de conférer à l'œuvre de Dante le prestige qui lui avait été nié jusqu'alors. La nécessité de différencier les deux textes amène à opposer à cet élément, typiquement italien, l'écriture bâtarde, utilisée traditionnellement pour la copie des textes en langue française; cela confère donc à la traduction une originalité qui lui est propre. Cette même originalité est aussi mise en exergue par l'apparat iconographique, réservé à la seule partie française ${ }^{76}$. Les dessins, qui opèrent une véritable synthèse visuelle du contenu de chaque chant, représentent une voie d'accès supplementaire au texte; le lecteur pouvait alors le lire en langue originale, en traduction et à travers les images, afin d'appréhender un texte considéré difficile et parfois obscur.

Un tout dernier aspect, qui n'a pas encore été pris en considération, est celui de la disposition des textes. Chaque tercet italien peut être identifié par l'adoption, de la part du copiste, d'une proéminence du premier vers ${ }^{77}$, tandis que pour la partie française le premier vers de chaque tercet est écrit en retrait. Cet effet graphique, contextualisé dans le cadre de tout ce que l'on vient d'illustrer, ne fait que démontrer que si les textes peuvent survivre séparément dans le manuscrit, ils peuvent aussi se lire de manière complémentaire. En utilisant les termes propres au gestaltisme, on pourrait affirmer que le postulat selon lequel «le tout est supérieur à la somme des parties» s'applique parfaitement à ce contexte; tout est étudié et pensé dans les plus petits détails: le choix de proposer une traduction très fidèle à l'original, l'esthétique de la clarté et de la lisibilité du texte, la mise en page, les écritures, leur calibre, et enfin leur disposition, tout concourt au résultat final. Le manuscrit conservant la première traduction française de l'Enfer de Dante est donc le lieu privilégié de la rencontre de deux cultures, italienne et française, différentes mais aussi complémentaires, qui peuvent exister séparément, mais dont les résultats, lorsqu'elles interagissent, peuvent être surprenants. Ce manuscrit mérite donc bien de prendre la place qui lui revient.

STEFANIA VIGNALI

Bergaigne, celle anonyme conservée dans le ms Wien, ÖNB 10201 et la traduction publiée par Grangier en 1596, le manuscrit Torino, BNU L.III.17, qui les précède, est le seul à présenter le texte italien en regard.

(73) Sur cette question cf. S. Vignali, De quelle source peut-on parler cit.

(74) Ce qui est prouvé par une imitation visiblement maladroite de cette écriture.

(75) Sur l'écriture humanistique en général voir B. Ullman, The origin and Development of Humanistic Script, Roma, Edizioni di Storia e Letteratura, 1960.
(76) Les dessins, exécutés à la plume avec quelques touches de bleu, représentent visuellement dans un seul cadre les passages les plus saillants du chant. Cette technique est définie par Renzi comme «rappresentazione continua» et «racconto secondo». L. RENZI, op. cit., p. 197.

(77) Ce qui ne reproduit pas la pagination de l'édition De Piasi, à partir de laquelle le texte italien présent dans le manuscrit fut copié. 